\title{
Prevalencia de resistencia de bacteria aisladas en hemocultivos, en un hospital universitario de Colombia
}

\author{
Prevalence of bacterial resistance isolated in hemocultives, in a university \\ hospital of Colombia
}

Marlene Duran-Lengua ${ }^{1}$, Luis Valladales-Restrepo² ${ }^{2}$ Roger Caraballo-Marimón ${ }^{3}$, Geraldine Romero Martínez ${ }^{4}$, Adrián Cabarcas-Tovar5, Cristina Bohórquez Moreno ${ }^{6}$

\section{Resumen}

Objetivo. Describir la frecuencia de los microorganismos y la resistencia antibiótica de bacterias aisladas en hemocultivos de pacientes con Bacteremia, en un hospital universitario de Colombia. Métodos. Se desarrolló un estudio observacional, descriptivo y de corte transversal, en individuos mayores de 18 ańos, en donde se describió los hemocultivos positivos, aislados en todos los servicios del Hospital Universitario del Caribe. Resultados. De los 211 hemocultivos analizados, el 53,1\% fueron hombres. Los microorganismos Gram positivos corresponden a 49,8\%, con una alta frecuencia de $S$. aureus en un $16,1 \%$. De los microorganismos Gram negativos fue aislado E.coli en un 18\%. La resistencia a vancomicina se estableció en 4,4\%. La $K$. pneumoniae presentó una resistencia a meropenem en un $15,3 \%$ de los casos. E.coli, P. aeruginosa y E.cloacae son sensibles a carbapénicos. Así en nuestro estudio las bacterias más frecuentemente aisladas en los hemocultivos fueron predominantemente Gram negativos, con resistencia a carbapénicos para algunas cepas de K. Pneumoniae.

\footnotetext{
1. Bacteriologa, Doctora en ciencias Básicas Biomédicas. Grupo de Investigación FARMABAC, Facultad de Medicina, Universidad de Cartagena, Cartagena, Colombia

Cvlac https://scienti.minciencias.gov.co/cvlac/visualizador/generarCurriculoCv.do?cod_rh $=0000729841$

ORCID: https://orcid.org/0000-0002-1104-6675

2. Medico Magister en Farmacología. Facultad de Medicina, Universidad de Cartagena, Cartagena, Colombia. Cvlac: https://scienti.minciencias.gov.co/cvlac/visualizador/generarCurriculoCv.do?cod_rh=0001384228 ORCID: https://orcid.org/0000-0002-4245-0101

3. Químico Farmacéutico, Magister en Farmacología. Universidad de Cartagena, Cartagena, Colombia. Cvlac: https://scienti.minciencias.gov.co/cvlac/visualizador/generarCurriculoCv.do?cod_rh=0000978736 ORCID: https://orcid.org/0000-0002-7386-0074

4. Médico. Grupo de Investigación FARMABAC, Facultad de Medicina, Universidad de Cartagena, Cartagena, Colombia. Cvlac: http://scienti.colciencias.gov.co:8081/cvlac/visualizador/generarCurriculoCv.do?cod_rh=0000051369 ORCID: https://orcid.org/0000-0003-1194-5504

5. Medico. Grupo de Investigación FARMABAC, Facultad de Medicina, Universidad de Cartagena, Cartagena, Colombia. Cvlac: https://scienti.minciencias.gov.co/cvlac/visualizador/generarCurriculoCv.do?cod_rh $=0001611758$ ORCID: https://orcid.org/0000-0002-7273-4113

6 Enfermera Msc farmacología. Grupo de Investigación FARMABAC, Facultad de Medicina, Universidad de Cartagena, Cartagena, Colombia.

Cvlac: https://scienti.minciencias.gov.co/cvlac/visualizador/generarCurriculoCv.do?cod_rh $=0001611758$ ORCID: https://orcid.org/0000-0002-3816-6749
} 
Palabras claves: bacteriemia, farmacorresistencia microbiana, pruebas de sensibilidad microbiana, antibacterianos.

\section{Abstract}

Objective. To describe the frequency of microorganisms and the antibiotic resistance of isolated bacteria in blood cultures of patients with bacteremia, in a university hospital in Colombia. Methods. An observational, descriptive, and cross-sectional study was developed in individuals older than 18 years, where the positive blood cultures were described, isolated in all the services of the University Hospital of the Caribbean. Results. Of the 211 blood cultures analyzed, $53.1 \%$ were men. The Gram-positive microorganisms correspond to $49.8 \%$, with a high frequency of S. aureus in $16.1 \%$. Of the Gram negative microorganisms, E.coli was isolated by $18 \%$. Vancomycin resistance was established at 4.4\%. K. pneumoniae showed resistance to meropenem in $15.3 \%$ of cases. E.coli, P. aeruginosa and E. cloacae are sensitive to carbapenes. Thus, in our study, the bacteria most frequently isolated in the blood cultures were predominantly Gram negative, with resistance to carbapenes for some strains of $K$. pneumoniae.

Keywords: bacteremia, drug resistance, microbial, drug microbial sensitivity tests, anti bacterial agents.

\section{Introducción}

La resistencia bacteriana ha sido asociada a la ineficaciadelos tratamientos farmacológicos establecidos para estos microorganismos, y repercutiendo de manera directa en un crecimiento proliferativo de infecciones con alta incidencia (1). En la actualidad la resistencia bacteriana es un problema global que afecta a muchas poblaciones y especialmente relacionadas con las instituciones de cuidado y atención de la salud de los pacientes. Las infecciones causadas por bacterias resistentes se asocian a una mayor morbi-mortalidad y aumentos en los costos de los tratamientos $(2,3)$; este problema se ha incrementado debido a prescripciones innecesarias o inadecuadas de antibióticos, debido a inoportunas pruebas diagnósticas, indicaciones en infecciones no requeridas o producto de la exposición microbiana a todos los antibióticos, ya sean por polimedicación $(4,5)$. El Foro Económico Mundial (WEF) ha establecido que posiblemente el mayor riesgo para la salud humana se presenta en forma de bacterias resistentes a los antibióticos, de este modo, se han incluidos 
estrategias para combatir la resistencia basándose en las prácticas tradicionales de control de infecciones, la administración de antibióticos y el desarrollo de nuevas terapias (6-8), para ello la Asamblea Mundial de la Salud aprobó en mayo de 2015 un plan de acción mundial sobre la resistencia a los antimicrobianos. Este plan contiene 5 objetivos, los cuales son: mejorar la sensibilización y los conocimientos en materia de resistencia a los antimicrobianos, reforzar la vigilancia y la investigación, reducir la incidencia de las infecciones, optimizar el uso de medicamentos antimicrobianos y asegurar que se realicen inversiones sostenibles en la lucha contra la resistencia a los antimicrobianos (7).

La resistencia a antibióticos varía geográfica y temporalmente en cuanto a su magnitud, este fenómeno es particular dependiendo directamente del tipo de antimicrobianos que adopte o implemente cada región, al igual que el tiempo que utiliza en cada protocolo de tratamiento realizado. Sin dejar de lado el perfil de resistencia adaptado en cada lugar (8).

Por esta razón es de gran importancia la implementación de sistemas de vigilancia y el desarrollo de líneas concretas para evaluar el impacto de las medidas de prevención y control de la resistencia a los antibióticos. Se estima que para 2050 las infecciones más comunes van a ser potencialmente mortales y las muertes anuales mundiales atribuibles a microorganismos resistentes podrían alcanzar los 10 millones de casos $(9,10)$. La OMS crea el Sistema Mundial de Vigilancia de la Resistencia a los Antimicrobianos que incluye actualmente a 52 países (25 de ingresos altos, 20 de ingresos medianos y siete de ingresos bajos). Asimismo, los Centros para el Control y la Prevención de Enfermedades estiman de manera conservadora que al menos 23.000 personas mueren anualmente en los Estados Unidos como resultado de una infección con un organismo resistente a los antibióticos y que más de 2 millones están en un alto riesgo $(11,12)$. Según un informe reciente del Reino Unido, el costo humano de la crisis de resistencia a los antibióticos se estima en 300 millones de muertes prematuras acumulativas para el año 2050, con una pérdida de hasta $\$ 100$ billones para la economía mundial (9).

Por lo tanto, se ha establecido amplios reportes que reconocen una alta resistencia en microorganismos relacionados con $E_{s}$ cherichia coli, Klebsiella pneumoniae, Staphylococcus aureus y Streptococcus pneumoniae, seguidas de Salmonella spp (5,13-15). Igualmente, entre los datos más importantes se puede destacar la significativa resistencia de la $E$. coli a las cefalosporinas de tercera generación y a las fluoroquinolonas en regiones de África, América, mediterráneo oriental, Europa, Asia Sudoriental y Pacífico Occidental (16-18). Además, la resistencia a meticilina hasta un $80 \%$ de las infecciones por $S$. aureus en áfrica y pacífico occidental, 90\% en América y más del $50 \%$ en las otras regiones mencionadas 
$(19,20)$. La resistencia de $K$. pneumoniae a las cefalosporinas de tercera generación también es elevada y generalizada en todas estas regiones (21).

En Colombia se ha notado un aumento en la resistencia bacteriana, el sistema nacional de vigilancia reportó 2.362 casos de resistencia antimicrobiana por infección asociada a dispositivos durante 2016, de los cuales $21,3 \%$ fallecieron. Por otro lado, en Cartagena no hay estudios que abarquen esta temática. Es por ello, que en el presente artículo se describe la frecuencia de microorganismos y la resistencia farmacológica de bacterias identificadas en hemocultivos de pacientes con bacteriemia en el Hospital Universitario del Caribe de Cartagena. El análisis continuo de la susceptibilidad a los antibióticos permite visualizar un panorama general de los perfiles de resistencia que presentan las bacterias, con la finalidad de predecir y establecer estrategias terapéuticas que orienten intervenciones farmacológicas más eficaces.

El empleo indiscriminado de los antimicrobianos que conduce a una rápida diseminación de cepas productoras de beta-lactamasas de espectro extendido, junto a la estadía hospitalaria prolongada de los pacientes, ha dado lugar a brotes importantes e incluso a la muerte del paciente. Un estudio realizado por Paterson y col reportó una tasa de mortalidad del 46\% de los pacientes, de 216 pacientes con bacteriemia por Klebsiella pneumoniae BLEE positiva (22).

\section{Material y método}

\section{Descripción de la población de estudio}

Estudio observacional, descriptivo y de corte transversal, llevado a cabo entre enero y diciembre de 2015. Se describió el total de hemocultivos positivos realizados en pacientes mayores de 18 años, en el Hospital Universitario del Caribe en Cartagena, Colombia. Se excluyeron los cultivos realizados en otros líquidos corporales diferentes a sangre, y aquellos hemocultivos con reportes en el antibiograma incompletos. En relación con esta última consideración los antibiogramas incompletos no fueron tenidos en cuenta para la descripción de la susceptibilidad antibiótica pero sí para caracterizar la frecuencia de los microorganismos aislados.

Adicionalmente se recolectaron los datos de identificación del paciente, género, servicio de aislamiento, microorganismo aislado, sensibilidad, y resistencia, los cuales fueron llevados a una base de datos en Microsoft Excel.

\section{Identificación y pruebas de susceptibilidad}

Se utilizaron métodos convencionales de identificación bioquímicas, conjunto con sistemas microbiológicos de detección empleando el panel MicroScan Pos Combo 34 (Beckman Coulter, USA), para bacterias 
Gram Positivas y el panel Neg/Urine Combo 60 (Beckman Coulter, USA), para Gram negativas. La susceptibilidad a los antimicrobianos fue interpretada en términos cualitativos (sensible o resistente) para facilitar la orientación terapéutica. Para los diferentes microrganismos se utilizaron los antimicrobianos y las especificaciones técnicas sugeridos por el Instituto de Estándares de Laboratorios Clínicos (CLSI).

Para el registro de la información, el estudio descriptivo de las variables estudiadas y la determinación del número $\left(\mathrm{N}^{\circ}\right)$ y porcentaje (\%) de cepas resistentes a los antimicrobianos, se utilizó el paquete estadístico IBM SPSS Statistics.

\section{Resultados}

Durante el período de enero a diciembre de 2015 en el Hospital Universitario del Caribe fueron reportados 211 hemocultivos positivos. De éstos, se excluyeron $8(3,79 \%)$, sólo para la descripción de los resultados de la resistencia a antimicrobianos, al no contar con la información completa del antibiograma, caso que ocurrió para el grupo de estreptococos. No se pudo obtener el total de hemocultivos realizados en este periodo de tiempo.

De los 211 reportes analizados, el 53,08 $\%$ fueron hombres. No se pudo analizar la variable edad, por no contar con dicha información. Se encontraron 22 especies bacterianas diferentes, de los cuales $36,36 \%$ $(\mathrm{n}=8)$ correspondían a bacterias Gram positivas mientras que el $63,63 \%$ estaban distribuidas en bacterias Gram negativas. Los microorganismos Gram positivos corresponden a 49,8\% $(\mathrm{n}=105)$ de los hemocultivos positivos, siendo el más frecuente el $S$. aureus con un $16,1 \%(n=34)$. Agrupando los Staphylococcus oagulasa Negativa (SCN), éstos corresponden al 26,5\% $(n=56)$, distribuidos en 11 especies diferentes. De los microorganismos Gram negativos el más frecuentemente aislado fue E.coli en un $18 \%(\mathrm{n}=38)$. En la tabla No 1 , se describe la frecuencia de los microorganismos aislados en los hemocultivos (Figura 1). 
Figura 1. Microorganismos aislados de hemocultivos. A. Bacterias Gram Positivas aisladas.

B. Bacterias Gram Negativas aisladas.

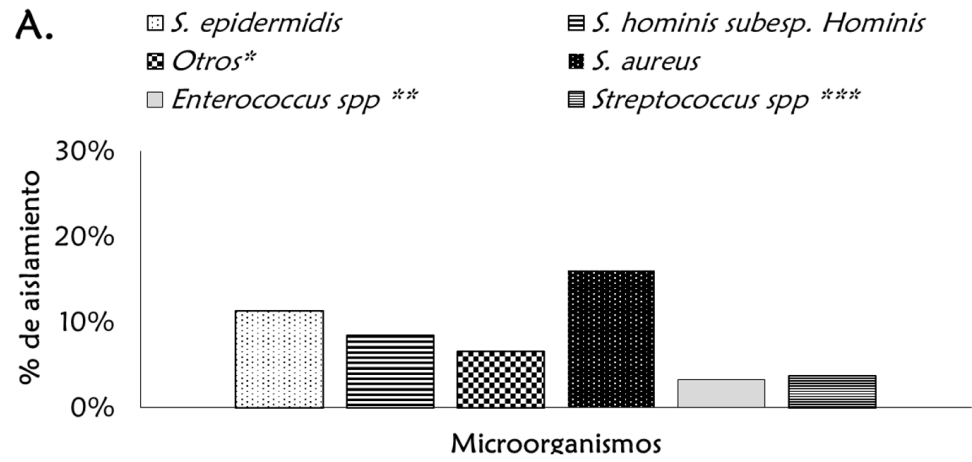

B.
ब $E$. coli
$\square$ K. pneumoniae
目 $P$. aeruginosa
$A$. baumannii/haemolyticus
E. cloacae
自 K. oxytoca
$\square$ A. Iwoffii
S Salmonella spp.
P. mirabilis
目E. brevis

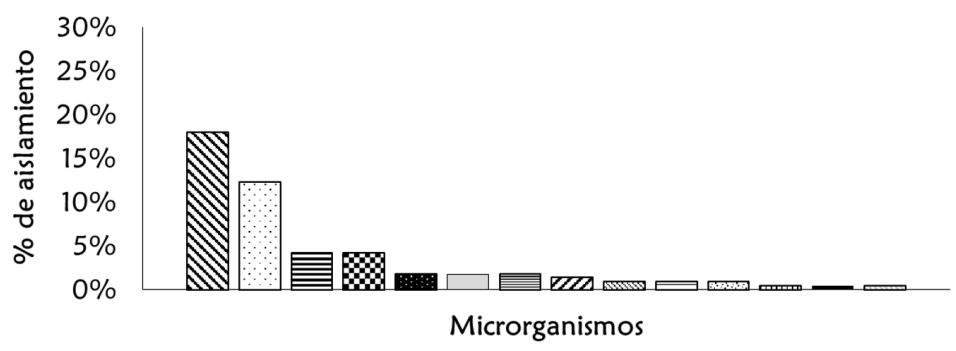

Fuente: Elaboración propia.

La resistencia hallada para el $S$. aureus fue de $29,4 \%$ para Cefazolina y Oxacilina. En cuanto a la clindamicina, la resistencia encontrada en el grupo de estafilococo coagulasa negativa fue cercana al 50\%, y sólo se halló un $S$. aureus que no era sensible.
La resistencia a vancomicina se documentó en $4,44 \%$ de los estafilococos, siendo sensibles todos los enterococos. Todos los gérmenes Gram positivos fueron resistentes a la ampicilina y sensibles a la daptomicina.

Tabla 1. Frecuencia de resistencia de las principales bacterias Gram positivas aisladas en hemocultivos.

\begin{tabular}{|c|c|c|c|c|}
\hline \multirow{3}{*}{$\begin{array}{l}\text { Agentes } \\
\text { antimicrobianos }\end{array}$} & \multicolumn{4}{|c|}{ Microorganismos } \\
\hline & & \multicolumn{3}{|c|}{ Staphylococcus coagulasa negativa } \\
\hline & $\begin{array}{l}\text { S. aureus } \\
n=34(\%)\end{array}$ & $\begin{array}{l}\text { S. epidermidis } \\
n=24(\%)\end{array}$ & $\begin{array}{l}\text { S. hominis subesp. Hominis } \\
n=18(\%)\end{array}$ & $\begin{array}{l}\text { Otros } \\
n=14(\%)\end{array}$ \\
\hline Penicilina & $33(97)$ & $24(100)$ & $16(88,8)$ & $14(100)$ \\
\hline Ampicilina & $34(100)$ & $24(100)$ & $18(100)$ & $14(100)$ \\
\hline
\end{tabular}




\begin{tabular}{|l|l|l|l|l|}
\hline \multirow{2}{*}{$\begin{array}{l}\text { Agentes } \\
\text { antimicrobianos }\end{array}$} & \multicolumn{3}{|c|}{ Microorganismos } \\
\cline { 2 - 5 } & $\begin{array}{l}\text { S. aureus } \\
n=34(\%)\end{array}$ & $\begin{array}{l}\text { S. epidermidis } \\
n=24(\%)\end{array}$ & $\begin{array}{l}\text { S. hominis subesp. Hominis } \\
n=18(\%)\end{array}$ & $\begin{array}{l}\text { Otros } \\
n=14(\%)\end{array}$ \\
\hline Ampicilina/Sulbactam & $10(29,4)$ & $15(62,5)$ & $9(50)$ & $13(92,8)$ \\
\hline Cefazolina & $10(29,4)$ & $15(62,5)$ & $9(50)$ & $13(92,8)$ \\
\hline Ciprofloxacina & $2(5,8)$ & $5(20,8)$ & $1(5,5)$ & $6(42,8)$ \\
\hline Clindamicina & $1(2,9)$ & $9(37,5)$ & $7(38,8)$ & $10(71,4)$ \\
\hline Daptomicina & 0 & 0 & 0 & 0 \\
\hline Eritromicina & $3(8,8)$ & $12(50)$ & $10(55,5)$ & $8(57,1)$ \\
\hline Trimetoprim/Sulfa & $1(2,9)$ & $6(25)$ & $6(33,3)$ & $3(21,4)$ \\
\hline Vancomicina & 0 & $0(0)$ & $1(5,5)$ & $2(14,2)$ \\
\hline Gentamicina & 0 & 0 & 0 & $6(42,8)$ \\
\hline Oxacilina & $10(29,4)$ & $15(62,5)$ & $9(50)$ & $13(92,8)$ \\
\hline Rifampicina & 0 & 0 & 0 & $1(7,1)$ \\
\hline Tetraciclina & $7(20,5)$ & $5(20,8)$ & $3(16,6)$ & $4(28,5)$ \\
\hline
\end{tabular}

Fuente: Elaboración propia.

El 86\% de los gérmenes Gram negativos presenta resistencia a la ampicilina. De las principales bacterias Gram negativas, solo el 2,43\% presentó resistencia a la amikacina mientras que el 23,17\% fue resistente a la gentamicina. Se encontró cepas de
$K$. pneumoniae con resistencia a meropenem y doripenem, con una frecuencia de $15,3 \%$ y $11,5 \%$ respectivamente. E.coli, P. aeruginosa y E.cloacae fueron sensibles a carbapénicos (Ver tabla 2).

Tabla 2. Frecuencia de resistencia de las principales bacterias Gram negativas aisladas en hemocultivos.

\begin{tabular}{|l|l|l|l|l|}
\hline \multirow{2}{*}{$\begin{array}{l}\text { Agentes } \\
\text { antimicrobianos }\end{array}$} & $\begin{array}{l}\text { E. coli } \\
n=38(\%)\end{array}$ & $\begin{array}{l}\text { K. pneumoniae } \\
n=26(\%)\end{array}$ & $\begin{array}{l}\text { P. aeruginosa } \\
n=9(\%)\end{array}$ & $\begin{array}{l}\text { E. cloacae } \\
n=9(\%)\end{array}$ \\
\hline Ampicilina & $31(81,5)$ & $26(100)$ & $9(100)$ & $8(88,8)$ \\
\hline Ampicilina Sulbactam & $13(34,2)$ & $11(42,3)$ & 0 & $5(55,5)$ \\
\hline Piperacilina/Tazobactam & 0 & $4(15,3)$ & $2(22,2)$ & $1(11,1)$ \\
\hline Cefazolina & $13(34,2)$ & $8(30,7)$ & 0 & $9(100)$ \\
\hline Cefepime & $10(26,3)$ & $7(26,9)$ & & $1(11,1)$ \\
\hline Cefotaxima & $11(28,9)$ & $7(26,9)$ & $3(33,3)$ & $1(11,1)$ \\
\hline
\end{tabular}




\begin{tabular}{|c|c|c|c|c|}
\hline \multirow{2}{*}{$\begin{array}{l}\text { Agentes } \\
\text { antimicrobianos }\end{array}$} & \multicolumn{4}{|c|}{ Microorganismos } \\
\hline & $\begin{array}{l}\text { E. coli } \\
n=38(\%)\end{array}$ & $\begin{array}{l}K . \text { pneumoniae } \\
n=26(\%)\end{array}$ & $\begin{array}{l}\text { P. aeruginosa } \\
n=9(\%)\end{array}$ & $\begin{array}{l}\text { E. cloacae } \\
n=9(\%)\end{array}$ \\
\hline Ceftazidima & $8(21)$ & $7(26,9)$ & 0 & $1(11,1)$ \\
\hline Cefoxitina & 0 & $2(7,6)$ & 0 & $7(77,7)$ \\
\hline Ceftriaxona & $11(28,9)$ & $6(23)$ & $4(44,4)$ & $1(11,1)$ \\
\hline Amikacina & 0 & $2(7,6)$ & 0 & 0 \\
\hline Gentamicina & $10(26,3)$ & $5(19,2)$ & $4(44,4)$ & 0 \\
\hline Tobramicina & $9(23,6)$ & $5(19,2)$ & $2(22,2)$ & $1(11,1)$ \\
\hline Ciprofloxacina & $15(39,4)$ & $6(23)$ & 0 & $2(22,2)$ \\
\hline Ertapenem & $1(2,6)$ & $4(15,3)$ & 0 & 0 \\
\hline Meropenem & 0 & $4(15,3)$ & 0 & 0 \\
\hline Doripenem & 0 & $3(11,5)$ & 0 & 0 \\
\hline Trimetoprim/Sulfa & $26(68,4)$ & $12(46,1)$ & 0 & $1(11,1)$ \\
\hline Aztreonam & $10(26,3)$ & $7(26,9)$ & 0 & $1(11,1)$ \\
\hline Tigeciclina & 0 & 0 & 0 & 0 \\
\hline
\end{tabular}

Fuente: Elaboración propia.

\section{Discusión}

Durante años el desarrollo de metodologías para la detección de bacterias en sangre juega un papel fundamental en el diagnóstico del paciente con diferentes manifestaciones clínicas, especialmente relacionados con el desarrollo de cuadros febriles dado que permite establecer la presencia de posibles infecciones, y puede conllevar al correcto seguimiento de terapias clínicas dirigidas a la elección correcta de los antibióticos según el resultado del antibiograma respectivo (23). Por tanto, el hemocultivo es una herramienta de gran valor para el seguimiento adecuado y diagnóstico eficaz. A pesar de que existen otras metodologías más fidedig- nas para la detección de bacterias aisladas, en la actualidad los hemocultivos son considerados herramientas de bajo costo que permiten realizar una indicación adecuada para la implementación de tratamientos cada vez direccionados $(24,25)$.

El presente estudio demostró que los microorganismos más frecuentemente aislados en hemocultivos en el Hospital Universitario del Caribe, correspondieron a bacterias Gram negativas (50,23\%), ligeramente superior sobre las Gram positivas, hallazgo que difiere del trabajo de Paz et al. $(26,27)$, los cuales evidenciaron la presencia principalmente de organismos Gram positivos $(67,6 \%)$. Asimismo, en el estudio se demostró que los microorganismos mayor- 
mente aislados fueron SCN (26,5\%), E.coli $(18 \%)$ y $S$. aureus $(16,1 \%)$, datos acordes al reporte de Paz et al. (27), quienes evidenciaron la detección de microorganismos Gram positivos y de igual modo demostrando variaciones en relación a la presencia de microorganismos Gram negativos, en donde el más frecuente fue $K$. pneumoniae, factor que puede estar relacionado con una amplia variación y diversidad en la microbiota de cada institución de salud $(28,29)$. De igual manera, de acuerdo con los SCN, se aislaron 11 especies diferentes, siendo los más frecuentes el $S$. epidermidis en un $42 \%$, similar a lo registrado por Fariña et al (30). En nuestro estudio el segundo más frecuente fue $S$. hominis sub esp. hominis con un $32,14 \%$, difiriendo marcadamente con estos autores, en donde la segunda especie más frecuente fue $S$. haemolyticus $(20,3 \%)$. Un comportamiento que fue dictaminado en las especies aisladas, indicando diferencias en la frecuencia de aparición. Estos contrastes epidemiológicos se podrían explicar debido a la diferencia geográfica y condiciones ambientales que podrían favorecer el desarrollo de algunas especies cuando se compararon ambos estudios (31).

Con respecto a los perfiles de resistencia de los antimicrobianos establecidos en nuestro estudio se ha indicado una alta ineficacia contra bacterias Gram positivas por parte de antibióticos de primera línea como penicilina, ampicilina, ampicilina/sulbactam y cefazolina $(32,33)$. Y un comportamiento medianamente resistente puede observarse por parte de clindamicina, eritromicina $y$ oxacilina (33). De este modo, se ha establecido dentro de los perfiles de resistencia microbiana un comportamiento particular con respecto a oxacilina, donde $S$. epidermidis fue reportada en $62,5 \%$, documentándose sensibilidad a vancomicina en todos los casos, hallazgos similares a lo encontrado por Fariña y cols, en donde además no se describió resistencia a vancomicina para ningún SCN, datos también encontrados por Paz et al. 26, sin embargo en nuestro estudio se hallaron 3 casos de resistencia a vancomicina (S. hominis subesp. hominis, S. xylosus y S. saprophyticus) (34). Por otro lado, se ha indicado que la resistencia por $S$. aureus a oxacilina en este estudio fue de 29,6\% (35$37)$; sin embargo nuestro hallazgo fue muy similar a lo descrito por Sader en América Latina, y ligeramente superior a la cohorte en México (38).

Por otra parte, en los antibiogramas resultantes para bacterias Gram negativas se ha observado que una notable resistencia a ampicilina y medianamente a ampicilina/ sulbactam conjunto a cefazolina y cefoxitina que resulto ser ineficaz contra las cepas de bacterias E. cloacae (39-41). De acuerdo a estos comportamientos evidenciados en bacterias Gram negativas, la resistencia encontrada en la mayoría de antimicrobianos para el E.coli es superior a lo descrito por Sader et al. (42). Lo que demuestra la pérdida progresiva de sensibilidad antimicrobiana a antibióticos de primera línea. La sensibilidad de $P$. aeruginosa a ceftazidime, cefepime y meropenem estuvo presente en todos los casos, a diferencia de lo reportado 
por otros autores. Lo que indica que este microorganismos no representa un problema de resistencia; a diferencia de lo encontrado para $K$. pneumoniae, en donde se aislaron cepas resistentes a meropenem en un $15,3 \%$, muy diferente a lo publicado por Sader en América Latina, en donde se reportó una sensibilidad del $99,8 \%$ a los carbapénicos $(43,44)$. Lo que indica la necesidad de fortalecer campañas de promoción para el uso racional de antibióticos y medidas de contención para evitar la selección y diseminación de cepas con estos fenotipos de resistencia, evitando brotes de infecciones intrahospitalarias, que representen una grave amenaza para la vida de los pacientes.

La resistencia a los antibióticos se considera uno de los problemas primordiales de salud pública en el mundo, ya que constituye un desafío tanto en el plano de la epidemiología como del tratamiento farmacológico de las enfermedades infecciosas. Podemos concluir en nuestro estudio que las bacterias más frecuentemente aisladas en los hemocultivos fueron bacterias Gram negativas, con resistencia a carbapénicos para algunas cepas de K. Pneumoniae; además de la poca sensibilidad de los SCN a oxacilina, y resistencia de algunas de ellas a vancomicina.

El grupo epidemiológico de infecciones de la institución, debe realizar de forma rutinaria estudios para determinar los perfiles de resistencia y sensibilidad de los diferentes microorganismo aislados en las diferentes pruebas de laboratorio, implementando mecanismos de vigilancia que permitan conocer de manera periódica y oportuna las tasas de resistencia antimicrobiana, ya que estas cambian en el tiempo, y con dicha información emplearla para el inicio de una adecuada terapia antimicrobiana empírica. Finalmente, se debe mejorar y procurar obtener la información de una forma más completa, para reducir así los sesgos y aportar un perfil epidemiológico más fidedigno.

\section{Referencias}

1. Lacoste J, Jarlier V, Coignard B, Vaux S. Estimating the morbidity and mortality associated with infections due to multidrug-resistant bacteria ( MDRB ),. Antimicrob Resist Infect Control [Internet]. 2016;5. Available from: http://dx.doi. org/10.1186/s13756-016-0154-z

2. Woolhouse M, Waugh C, Perry MR, Nair H. Global disease burden due to antibiotic resistance - state of the evidence. J Glob Health. 2016;6:010306.

3. Kajumbula H, Fujita AW, Mbabazi O, Najjuka C, Izale C, Akampurira A, et al. Antimicrobial Drug Resistance in Blood Culture Isolates at a Tertiary Hospital, Uganda. Emerg Infect Dis. 2018;24:174-5.

4. Constanza L, Antolinez D, Bohorquez A, Corredor A. Bacterias anaerobias: procesos que realizan y contribuyen a la sostenibilidad de la vida en el planeta. Nova.2015; 13(24): 55-82.

5. Fair RJ, Tor Y. Antibiotics and Bacterial Resistance in the 21st Century. Perspect Medicin Chem. 2014;6:25-64.

6. Worthington RJ, Melander C. Combination Approaches to Combat Multi-Drug Resistant Bacteria. Trends Biotechnol. 2014;31:177-84.

7. WHO. Plan de acción mundial sobre la resistencia a los antimicrobianos. Geneva, Switzerland: WHO Document Production Services; 2016. p. 1-30. 
8. Powers JH, Evans SR, Aaron S. Studying new antibiotics for multidrug resistant infections : are today' $s$ patients paying for unproved future benefits? BMJ. 2018;360:1-6.

9. González E, Zapata A, Sánchez-Henao D, Chávez-Vivas M. Resistencia a antibióticos $\beta$-lactámicos y eritromicina en bacterias de la cavidad oral. Nova. 2020; 18(34): 27-45.

10. Cassini A, Högberg LD, Plachouras D, Quattrocchi A, Hoxha A, Simonsen GS. Attributable deaths and disability-adjusted life-years caused by infections with antibiotic-resistant bacteria in the EU and the European Economic Area in 2015: a population-level modelling analysis. Lancet Infect Dis. 2019;19:56-66.

11. WHO. Worldwide country situation analysis: Worldwide country situation analysis : [Internet]. Geneva, Switzerland: WHO Press; 2015. p. 1-43. Available from: www.who.int/drugresistance/en/

12. WHO. Antimicrobial Resistance [Internet]. Copenhagen: World Health Organization Regional Office for Europe; 2017. p. 1-7. Available from: www.euro.who.int/sdgs (C)

13. Rasheed MU, Thajuddin N, Ahamed P, Teklemariam Z, Jamil K. ANTIMICROBIAL DRUG RESISTANCE IN STRAINS OF Escherichia coli ISOLATED FROM FOOD SOURCES. Rev Inst Med Trop Sao Paulo. 2014;56:341-6.

14. Farrell DJ, Castanheira M, Mendes RE, Sader HS, Jones RN. In Vitro Activity of Ceftaroline Against Multidrug-Resistant Staphylococcus aureus and Streptococcus pneumoniae: A Review of Published Studies and the AWARE Surveillance Program ( $2008-2010$ ). 2012;55:206-14.

15. Reiss-mandel A, Regev-yochay G. Staphylococcus aureus and Streptococcus pneumoniae interaction and response to pneumococcal vaccination: Myth or reality ? 2016;351-7.

16. González C, Mendoza-medellín A. Resistencia a cefalosporinas de tercera y cuarta generación en enterobacterias productoras de infecciones nosocomiales y caracterización preliminar de los plásmidos involucrados. Cienc ergo sum. 2008;15:83-90.
17. Karczmarczyk M, Martins M, Quinn T, Leonard N. Mechanisms of Fluoroquinolone Resistance in Escherichia coli Isolates from Food-Producing Animals. Appl Environ Microbiol. 2011;77:7113-20.

18. Rath S, Sc M, Padhy RN, Ph D. Prevalence of fluoroquinolone resistance in Escherichia coli in an Indian teaching hospital and adjoining communities. J Taibah Univ Med Sci [Internet]. 2015;10:504-8. Available from: http://dx.doi.org/10.1016/j.jtumed.2015.02.009

19. Abdulgader SM, Shittu AO, Nicol MP, Kaba M. Molecular epidemiology of Methicillin-resistant Staphylococcus aureus in Africa : a systematic review. Front Microbiol. 2015;6:348.

20. Lee AS, Lencastre H De, Garau J, Kluytmans J, Malhotra-kumar S, Peschel A, et al. Methicillin-resistant Staphylococcus aureus. Nat Publ Gr [Internet]. 2018;4:1-23. Available from: http://dx.doi. org/10.1038/nrdp.2018.33

21. Lee S, Han SW, Kim KW, Song DY, Kwon KT. Third-generation cephalosporin resistance of community-onset Escherichia coli and Klebsiella pneumoniae bacteremia in a secondary hospital. Korean J Int Med. 2014;29:49-56.

22. Paterson D, Mulazimoglu L, Casellas J. Epidemiology of ciprofloxacin resistance and its relationship to extended-spectrum B-lactamase producing in Klebsiella pneumoniae isolates causing bacteriemia. Clin Infect Dis. 2000; 30: 473-478.

23. Elshout G, Monteny M, Wouden JC Van Der, Koes BW, Berger MY. Duration of fever and serious bacterial infections in children: a systematic review. BMC Fam Pract [Internet]. 2011;12:33. Available from: http://www.biomedcentral.com/1471$2296 / 12 / 33$

24. Pien BC, Sundaram P, Raoof N, Costa SF, Mirrett $S$. The Clinical and Prognostic Importance of Positive Blood. AJM [Internet]. 2010;123:819-28. Available from: http://dx.doi.org/10.1016/j.amjmed.2010.03.021 
25. Snyder JW. Blood Cultures: the Importance of Meeting Pre-Analytical Requirements in Reducing Contamination, Optimizing Sensitivity of Detection, and Clinical Relevance. Clin Microbiol Newsl [Internet]. 2015;37:53-7. Available from: http:// dx.doi.org/10.1016/j.clinmicnews.2015.03.001

26. Maldonado NA, Múnera MI, López JA, Sierra P, Robledo C, Robledo J, et al. Tendencias de la resistencia a antibióticos en Medellín y en los municipios del área metropolitana entre 2007 y 2012 : resultados de seis años de vigilancia. Biomédica. 2014;34:433-46.

27. Paz-Montes, América Fuenmayor-Boscán A, Sandrea-Toledo L, Pińa-Reyes E, López-Dávila M, Navarro-López P. Incidencia de microorganismos en hemocultivos procesados en un hospital del estado Zulia y su resistencia a los agentes antimicrobianos Incidence of Microorganisms in Blood Cultures Processed at a Hospital in the State of Zulia and Resistance. Kasmera. 2015;43:16-33.

28. Navon-venezia S, Kondratyeva K, Carattoli A. Klebsiella pneumoniae : a major worldwide source and shuttle for antibiotic resistance. FEMS Microbiol Rev. 2017;41:252-75.

29. Martin RM, Bachman MA. Colonization, Infection, and the Accessory Genome of Klebsiella pneumoniae. Front Cell Infect Microbiol. 2018;8:4.

30. Farińa N, Carpinelli L, Samudio M, Guillén R, Laspina F, Sanabria R, et al. Staphylococcus coagulasa-negativa clínicamente significativos. Especies más frecuentes y factores de virulencia. Rev Chil infectología. 2013;30:480-8.

31. Xu J, Yan C, Wang L, Zhou Q. Changes of Antimicrobial Resistance among Coagulase-Negative Staphylococci Isolated in 8 Consecutive Years in the First Bethune Hospital. Phys Procedia [Internet]. 2012;33:1190-3. Available from: http://dx.doi.org/10.1016/j.phpro.2012.05.195

32. Duarte-Raya F, Granados-Ramírez MP. Resistencia antimicrobiana de bacterias en un hospital de tercer nivel. Rev Med Inst Mex Seguro Soc. 2012;50:289-300.
33. Lazo-Arispe GF, Mamani -Flores E, Vargas-Loroño E, Camacho-Aguilar JR, Sahonero-Ampuero O. Sensibilidad y resistencia en el antibiograma del Staphylococcus aureus en pacientes del Hospital Clinico Viedma. Rev Científica Cienc Médica. 2013;16:15-7.

34. El-zawahry Y, Abdel-shafi S, Zaki M, El-serwy H. Phenotypic and Genotypic Investigation of Methicillin Resistant Staphylococci Species Isolated from Children with Sepsis in Egypt. Egypt J Bot. 2018;58:11-22.

35. Jeong J, Chang CL, Park TS, Lee SH, Kim SR, Jeong SH. Early Screening of Oxacillin-Resistant Staphylococcus aureus and Staphylococcus epidermidis from Blood Culture. J Korean Med Sci. 2002;17:168-72.

36. Bennett K, Sharp SE. Rapid Differentiation of Methicillin-Resistant Staphylococcus aureus and Methicillin-Susceptible Staphylococcus aureus from Blood Cultures by Use of a Direct Cefoxitin Disk Diffusion Test. J Clin Microbiol. 2008;46:3836-8.

37. Walter J, Noll I, Feig M, Weiss B, Claus H, Werner $G$, et al. Decline in the proportion of methicillin resistance among Staphylococcus aureus isolates from non-invasive samples and in outpatient settings, and changes in the co- resistance profiles: an analysis of data collected within the Antimicrobial Resistance. BMC Infect Dis. 2017;17:169.

38. Adame-g R, Toribio-jimenez J, Vences-velazquez A, Rodr E, Cristina M, Dionisio S, et al. Methicillin-Resistant Staphylococcus aureus (MRSA) in Artisanal Cheeses in México. Int J Microbiol. 2018;2018:6.

39. Famiglietti $A$, Quinteros $M$, Vázquez $M$, Marín M, Nicola F, Radice M, et al. Consenso sobre las pruebas de sensibilidad a los antimicrobianos en Enterobacteriaceae. Rev Argent Microbiol. 2005;37:57-66.

40. Turnidge J. Cefazolin and Enterobacteriaceae: Rationale for Revised Susceptibility Testing Breakpoints. Med Microbiol. 2011;52:917-24. 
41. Gutiérrez Lesmes O. Revista CUIDARTE. Rev Cuid. 2015;6:947-54.

42. Sader HS, Jones RN, Andrade-baiocchi S, Biedenbach DJ, Sentry T, Group P, et al. Four-year evaluation of frequency of occurrence and antimicrobial susceptibility patterns of bacteria from bloodstream infections in Latin American medical centers. Diagn Microbiol Infect Dis. 2003;44:273-80.

43. Velásquez J, Hernández R, Pamo O, Candiotti M, Pinedo Y, Sacsaquispe R, et al. Klebsiella pneumoniae resistente a los carbapenemes. Primer caso de carbapenemasa tipo KPC en Perú. Rev Soc Peru Med Interna. 2013;26:192-6.

44. Echeverri-toro LM, Rueda Z V, Maya W, Agudelo Y, Ospina S. Klebsiella pneumoniae. Rev Chil infectología. 2010;29:175-82. 